\title{
Cutting Mechanism Study of an Innovative Non-Planar Cutter PDC Bit and Its Field Application
}

\author{
Ning Li ${ }^{1}$, Xueqing Teng ${ }^{1}$, Hongtao Liu ${ }^{1}$, Duanrui Zhang ${ }^{1}$, Bo Zhou ${ }^{1}$, Li Zhao ${ }^{1}$, Renqing He ${ }^{1}$, \\ Jinsong Guo ${ }^{2}$ \\ ${ }^{1}$ PetroChina Tarim Oilfield Company, Korla, Xinjiang, China \\ ${ }^{2}$ Petro China, Bohai Drilling Engineering Company, Directional Drilling Branch, Tianjin, China

\section{Email address:} \\ lining-tlm@petrochina.com.cn (Ning Li), tengxq-tlm@petrochina.com.cn (Xueqing Teng), liuhongtao-tlm@petrochina.com.cn (Hongtao Liu), \\ zhangdr-tlm@petrochina.com.cn (Duanrui Zhang), zhoubo-tlm@petrochina.com.cn (Bo Zhou), zhaoli-tlm@petrochina.com.cn (Li Zhao), \\ hrq-tlm@petrochina.com.cn (Renqing He), 29194954@qq.com (Jinsong Guo) \\ ${ }^{*}$ Corresponding author
}

\section{To cite this article:}

Ning Li, Xueqing Teng, Hongtao Liu, Duanrui Zhang, Bo Zhou, Li Zhao, Renqing He, Jinsong Guo. Cutting Mechanism Study of an Innovative Non-Planar Cutter PDC Bit and Its Field Application. International Journal of Oil, Gas and Coal Engineering.

Vol. 8, No. 3, 2020, pp. 53-57. doi: 10.11648/j.ogce.20200803.11

Received: April 16, 2020; Accepted: May 7, 2020; Published: May 28, 2020

\begin{abstract}
Tarim Kuqa tight sandstone reservoir is overlaid with extreme thick conglomerate, which has high compressive strength, strong heterogeneity and poor drill ability, resulting in low drilling ROP and less bit drilling footage. Through research study and analysis of PDC bit failure and wear modes, this paper described the development of an innovative non-planar PDC bit through cutting mechanism study and lab testing verification. When drilling through the strong homogeneity formation, it will still be surface contact between the non-planar cutter and the rock and therefore the rock-breaking mechanism is still the conventional shearing fracturing; When drilling through the heterogeneity formation, the convex ridges will generate the linear contact with the hard rock and the mechanical stress will be accumulated at the conglomerate surface to initially create the cracking and then break the rock with composite cutting mechanism of shearing and crushing to improve the rock breaking efficiency. Experimental results also showed that the non-planar cutter delivers several folds of impact resistance improvement over conventional cutter. The bit was successfully field tested in ultra-thick conglomerate layer in the Kuqa foreland thrust belt of the Tarim basin. The field test tripled bit footage and doubled ROP comparing to same intervals and formation in offset wells. It demonstrated the broader prospect of the non-planar PDC technology in conglomerate and highly heterogeneous applications.
\end{abstract}

Keywords: Non-Planar Cutter, PDC Bit, Conglomerate Drilling, Performance Improvement, Tarim Oilfield

\section{Introduction}

Kelasu structural belt lies in the northern Kuqa depression of Tarim basin and the overall distribution is nearly East-West direction. It's been divided into Awate block, Bozi block, Dabei-tubei block, Keshen block and Kela block by the transformation zone from west to east [1-3]. In 2004, an appraisal well was drilled in Tarim Bozi block with the target interval of Cretaceous Bashijiqike (K1bs) formation. Roller cone bit was used for the whole well to drill through the conglomerate, however, the rate of penetration was very low and it took 397 days in total to complete. Two more wells were drilled afterwards with continuous circulation air drilling [4-5] implemented to improve the performance and the actual rate of penetration (ROP) has improved significantly. However, it will result in the high risk of borehole collapse. One of wells with continuous circulation air drilling was stuck after drilling to total depth at $3502 \mathrm{~m}$ while tripping to $3018 \mathrm{~m}$. The drill string dropped into the hole after explosion for break-outing and it took one month for remedial solution solving the complicated downhole situation, and sidetracking had to be conducted in the end. The bottom hole assembly (BHA) of impregnated bits and high-speed turbine [6-7] is another effective method of 
drilling in conglomerate. It is been implemented in this block for performance improvement and the average ROP has been doubled. The disadvantage of this BHA is: first, the normal turbine BHA will be a building-up assembly due to the formation dip in the upper section of Bozi block. Therefore, the total footage will be affected by the turbine instability, measurement while drilling tools (MWD) failure rate and frequent directional correction. Secondly, the rent cost of impregnated bit and turbine will be 2-3 times of that of Polycrystalline Diamond Compact (PDC) bit, which will result in higher drilling cost. From the wells drilling summary, the main issues for drilling ROP improvement in Bozi block will be limited tool for upper thick conglomerate, long drilling period and high cost [8]. Therefore, how to improve the drilling efficiency in thick conglomerate and reduce drilling days has become an urgent issue to be resolved for the exploration and development in Bozi block.

\section{An Innovative Non-Planar Cutter PDC Bit Design and Composite Cutting Mechanism}

\subsection{PDC Bit Implementation and Failure Analysis}

When drilling through strong heterogeneity formation, the performance of conventional PDC bit will normally be low ROP, short bit footage, frequent tripping and high drilling cost. The main reason would be that the conventional PDC bit cutters are cylindrical and the contact area is normally located in the partial front-end area, therefore, the impact force to the cutters will be introduced during the high-speed drilling through the conglomerate.
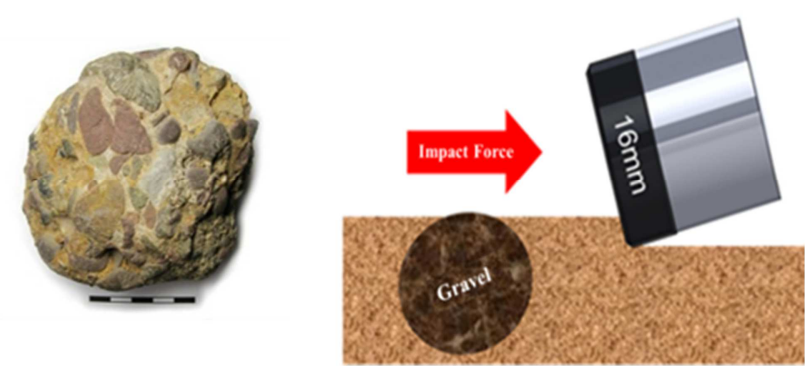

Figure 1. Conglomerate sample and the example of PDC cutter impacted by the conglomerate.

In addition, during the cooling process after the sintering in HPHT press, the contraction rate of hard alloy is faster than that of PDC material due to the different coefficient of thermal expansion [9-11], which results in high residual stress in the PDC layer. Furthermore, based on the different geometry design of the hard alloy [12-13], the generated residual stress at the top edge is normally demonstrated as different level of tensile stress. As shown in figure 2, the tensile stress will overlap with the facial impact force when drilling through the conglomerate, which increased the damage severity of PDC cutters.

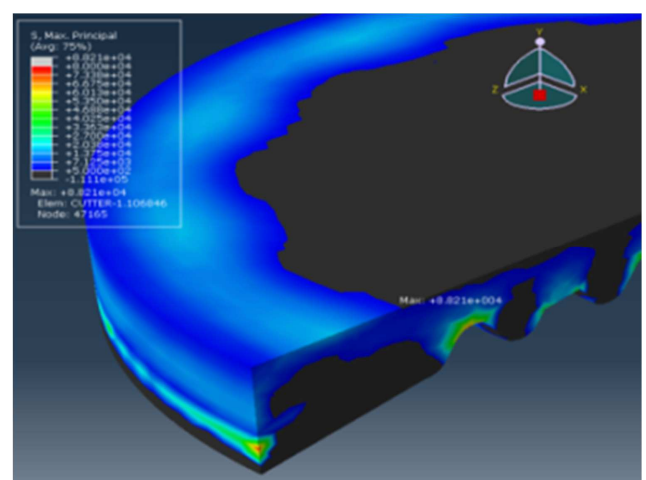

Figure 2. Tensile stress distributions at the edge of PDC cutter with finite element simulation.

Therefore, the impact force from the formation along the cutting direction could be seen clearly when drilling through the conglomerate or high heterogeneity formation, and consequently the fracture surface would be introduced at the PDC cutter edge. As shown in figure 3, the fracture surface from the PDC layer along the hard alloy matrix will produce crack extension [14-15] and the original cutting edge will be lost.

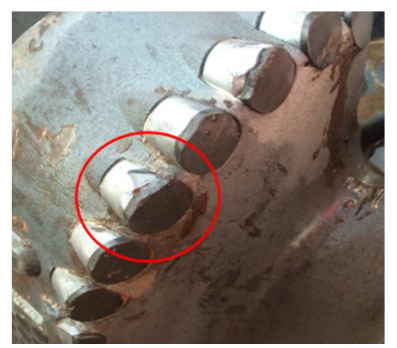

Figure 3. Conventional PDC bit cutter impact damage.

\subsection{Non-Planar PDC Cutter Design}

From the PDC cutter and bit failure mode [16], it can be concluded that the key factor to drill through the conglomerate effectively is to improve the cutter capability of the facial impact [17]. Therefore, an innovative non-planar cutter and its corresponding PDC bit design have been proposed. In this non-planar cutter design, the interaction area between cutter and formation has changed from plane to three dimensions non-planar, and the top PDC edge is divided into three inclined surfaces, separated by three convex ridges, and another new surface in the middle. As shown from figure 4, during the cutter distribution, place one convex ridge at the cutting edge as the tool face of the bit.

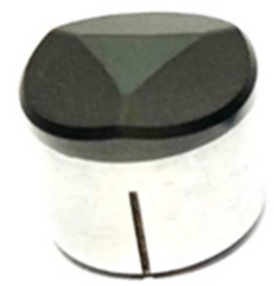

Figure 4. Innovative non-planar cutter design. 


\subsection{Non-Planar Cutter Composite Cutting Mechanism}

With this new cutting structure, the conglomerate will generate the linear contact with the convex ridges, instead of surface contact with the diamond, as shown in figure 5. The mechanical stress will be accumulated at the conglomerate surface due to the conglomerate extruding by the convex ridges, therefore the formation will initially create the cracking [18] and then break. In the later period of bit operation, the convex ridge edges are completely grounded due to effect between the formation and the cutting edge. Then the triangular plane of the cutter starts to contact with the formation, thus forming a new cutting edge to continue shearing the formation.

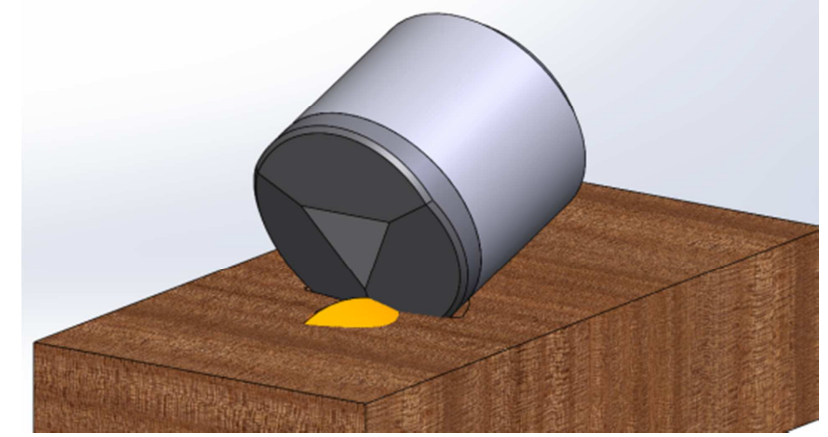

Figure 5. Conglomerate breaking by convex ridge extruding when cutting with non-planar cutters.

When drilling through the strong homogeneity formation, e.g. sandstone and mudstone, it will still be surface contact between the non-planar cutter and the rock. The rock-breaking mechanism is still the conventional shearing fracturing [19]; therefore, there is no difference for the rock-breaking pattern between the non-planar and planar cutters. However, the cutting edge distributed at the side of the convex ridge is shorter than the conventional cutter, so the generated cutting will be more dispersed. If the dispersed cuttings are accumulated at the top of the cutters, it's possible to cause bit balling. The surface has been divided into three inclined surfaces, which will create the lateral and forward forces to prevent the cuttings from accumulating at the top of the cutters, and eventually avoid the bit balling.

\section{Non-Planar PDC Cutter Laboratory Test}

After the design finalization for the non-planar cutters, laboratory tests for each performance indexes shall be conducted. When drilling through the heterogeneous formations, the contact between the cutter and the conglomerate will be linear [20], therefore the PDC convex ridge is subjected to the force from the formation. Hence the lab test for the positive impact force shall be conducted. The test method is to place the cutters in a specially designed fixture and strike the cutting ridge in a certain force and frequency in order to simulate the downhole situation of conglomerate cutting. The force and frequency could be adjusted during the test; thereby the actual working situation could be modelled at the maximum possibility [21]. If the impact times exceed the threshold number, it could be considered to pass. The comparison groups are the conventional planar cutters with the same material. From the result, the impact resistance capability of the non-planar cutters is far better than that of the planar ones. All the non-planar cutter samples passed the impact test; however, the planar cutters will normally fail at $10 \%$ of the threshold number. This test has proved that the impact resistance capability of the non-planar cutters has improved several times comparing to the conventional planar cutters.
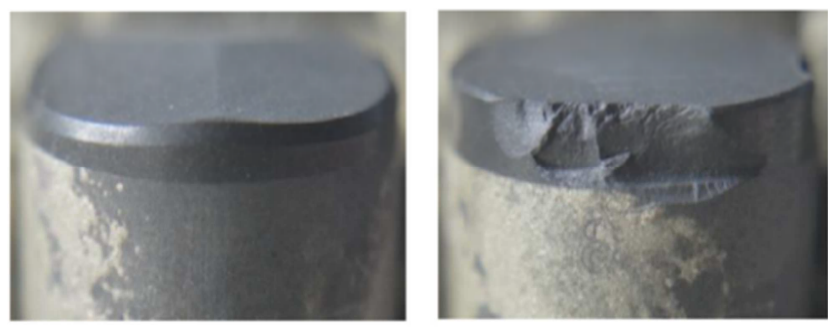

Figure 6. Damage comparisons of impact test samples.

When drilling through the conglomerate, quite amount of sandstone and mudstone will normally encounter. Laboratory test shall be conducted to verify if the rock-breaking efficiency of the non-planar cutters is affected in the homogeneity formation. Therefore, the second test is the rock-breaking efficiency test for the single cutter under pressure. During the test, the sample cutter will be welded onto the specific fixture and placed in a pressured chamber. The pressure will be provided by the oil. The sandstone sample with the compressive strength $110 \mathrm{MPa}$ will be placed a rotated in the pressured chamber. The cutters will shear the rock with given cutting depth and the forces from three directions during the cutting will be monitored by three-axis sensors. The non-planar cutters and the comparative planar cutters will conduct the cutting test under the same condition. After several comparison tests, the result shows no significant difference between the non-planar and planar cutters regarding to the cutting efficiency. Under the condition of breaking through the same volume of sandstone, the required axial force (vertical direction) and shearing force (cutting direction) will be at the same level as the planar cutters, which indicates the similar cutting efficiency between the non-planar cutters and conventional planar cutters when drilling through the homogeneity formation (e.g. sandstone, mudstone etc.). 

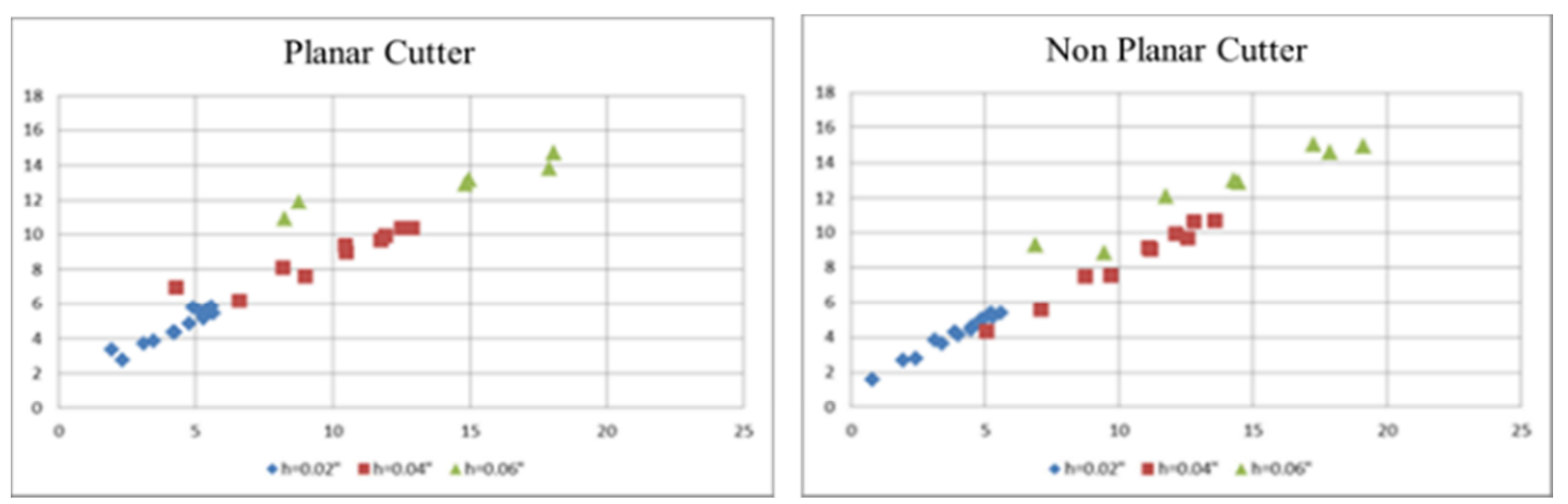

Figure 7. Cutting efficiency comparison with Conventional cutter.

\section{Non-Planar PDC Cutter Bit Field Test}

The field test well is located in Bozi block and the designed total depth is $8090 \mathrm{~m}$. The completion zone is Cretaceous Baxigai formation and the target zone is Cretaceous Bashijiqike (K1bs) formation. The formation to be drilled from the top to the bottom will respectively be Quaternary Xiyu formation (the main lithology is medium thick very thick gravel and fine conglomerate, medium thick very thick layered sandy conglomerate), upper tertiary Kuqa formation (the main lithology for the upper section is medium $\sim$ thick layered fine-grained conglomerate, small conglomerate and sandy conglomerate; the main lithology for the middle and lower section is medium thick layered conglomerate, sandy conglomerate, medium sandstone with conglomerate, fine sandstone with conglomerate), Kangcun formation (the main lithology is medium thick very thick layered fine sandstone with conglomerate, interbedded with different thickness of small conglomerate), Jidike formation, PalaeogeneSuweiyi formation, Kumugeliemu formation, Cretaceous Bashijiqike formation and Baxigai formation. In conclusion, the formations above Jidike are all containing conglomerate.

The conventional PDC bit has short drill footage and frequent tripping due to the early damage under the impact of conglomerate. On account of the characteristic of the conglomerate interval, in order to achieve and maintain the high ROP, bit type of high aggression 17 1/2" MT616X with six blades and dual rows of teeth has developed with the non-planar cutters distributed on the cutting structure. The field test was conducted in August 2019.

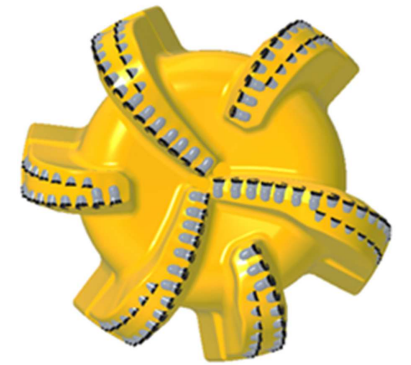

Figure 8. Non-planar bit design of 17 1/2" MT616X.
The non-planar PDC bit 17 1/2" MT616X was run in hole on 22 August 2019 with the initial depth of $2613 \mathrm{~m}$ and drilled to $2946 \mathrm{~m}$ with the actual drilling time 163 hours, drilling footage $333 \mathrm{~m}$ and average ROP $2.04 \mathrm{~m} / \mathrm{hr}$. The lithology for the formation to be drilled is mainly varicolored small conglomerate and fine-grained conglomerate with igneous rock and limestone interbedded. The international association of drilling contractors (IADC) dull grading is 1-3-BT-S-X-X-X-CT-TD.
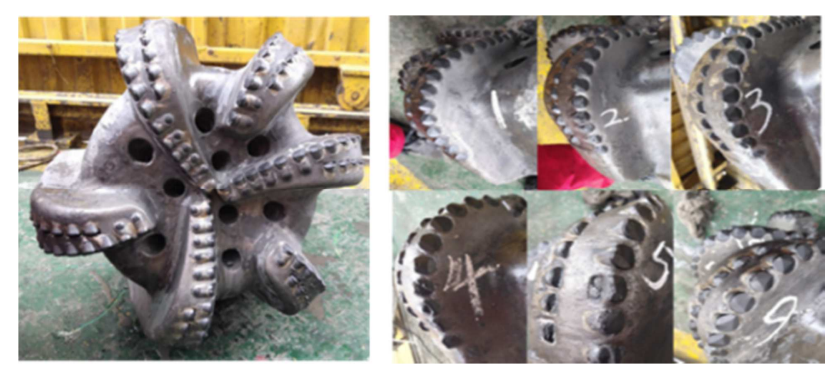

Figure 9. Bits pulled-out pictures of 17 1/2" MT616X.

When drilling though the conglomerate, the lithology of the cutting returns includes quartz gravel and metamorphic gravel. The returned cuttings indicate that the convex ridge of the non-planar cutters has indeed generated the concentrated stress to extrude and crush the rocks, and the cracking on the crushing surface extends along and creates the flakes cuttings. When drilling though the sandstone and mudstone formation, due to the homogeneity and plasticity of the sandstone and mudstone, the cuttings produced by the non-planar cutter PDC bit are similar to the planar cutter bit as the strip form. The only difference is that there will be a groove in the middle of the cutting due to the presence of the convex ridge.

Compared with the bit data of offset wells from the same zone in the same block, it shows clearly that the footage and ROP of the non-planar PDC bit with significant performance improvement by tripled bit footage and doubled ROP. ROP improvement is the most distinct with low risk of downhole complicated situations and lowest integrated drilling cost, which will play the actual role of reducing cost and increasing benefits. 


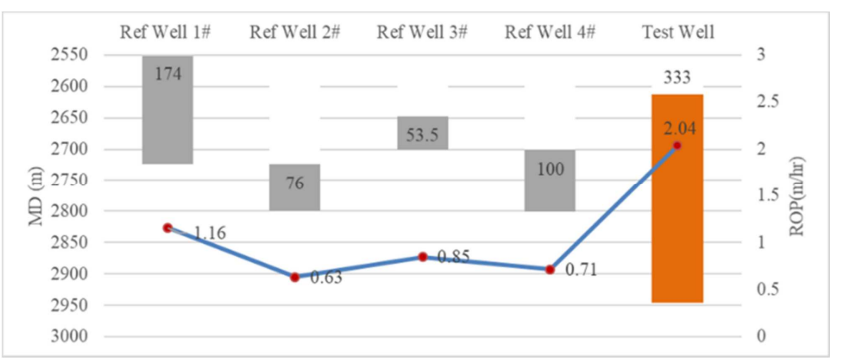

Figure 10. Bit performance comparison with offset wells.

\section{Conclusion}

Non-planar cutters with three inclined surfaces were adopted in the innovative non-planar PDC bit, which extrudes and breaks the strong heterogeneity formation with convex ridge while breaking the homogeneity formation by means of shearing, therefore the composite rock-breaking mechanism was established. Laboratory test has proved that the capability of impact resistance has improved more than ten times comparing with the conventional PDC bit. With non-planar PDC bits field tests conducted in the upper conglomerate formation of Bozi block, the drilling performance has been improved by tripled bit footage and doubled ROP comparing to the offset wells with similar lithologies. Practice has proved that the composite rock-breaking mechanism of the non-planar cutters can be well applied in the strong heterogeneity formation for better drilling performance.

\section{Acknowledgements}

The authors wish to express their gratitude to management at Petro China Tarim Oilfield Company and Gree Energy Services Inc for the permission to release the field performance, well data and bit development process.

\section{References}

[1] LEI G, XIE H, ZHANG J, et al. Structural Features and Natural Gas Exploration in the Kelasu Structural BeleKuq Depression [J]. Oil \& Gas Geology, 2007 (06): 816-820+835.

[2] WANG Z., ZHONG D., SUN H., et al. Sedimentary Characteristics and Distribution of Neogene-Quaternary Gravel Layers in the Middle of Ku Depression, Tarim Basin [J]. ACTASedimentologica Sinica, 2013, 31 (02): 282-290.

[3] LIL., LIU Y., LIU X., et al. Distribution Characteristics of Neogene-quaternary Gravel Layers in Western Kelasu Structural Belt Tarim Basin [J]. Henan Science, 2019, 37 (10): 1659-1664.

[4] ZHENG Lihui, LIU Hao, ZENG Hao, et al. Zhang Wenchang $\&$ Wang Chao. Evaluation of working fluid damage in fractured reservoirs using flow rate instead of permeability, [J]. Natural Gas Industry, 2019, 39 (12): 75-80.

[5] ZHENG Lihui, LI Xiuyun, SU Guandong, et al. pplicability of working fluid damage assessment methods for coalbed methane reservoirs. [J]. Natural Gas Industry, 2018, 38 (9):
28-39.

[6] MAHongyan, KONG Fanggang, YANG Jinsheng, et al. Sidetracking technique of slim hole in AT-6X well of ultra-deep and ultra-high temperature buried hill gas well. [J/OL]. Oil Drilling\&Production Technology, 1-11 [2020-03-29]. http://kns.cnki.net/kcms/detail/13.1072.TE.20200103.1812.00 4.html.

[7] WANGS., WANG W., YU S. Combination of turbodrill and impregnated diamond bit to enhance ROP in drilling of wells in piedmont Zone, Tarim Basin [J]. Oil Drilling \& Production Technology. 2016, 38 (02): 156-159.

[8] QIAO Y., BIAN P., LUO F. Application of High-Speed Turbodrilling Technology in Tarim Keshen Gas Field [J]. Drilling \& Production Technology, 2014, 37 (04): 30-32+2.

[9] DOU L., LI T., ZHANG M., et al. Study on Techniques and Application of New Bits for Difficult Formation in Piedmont Region [J]. China Petroleum Machinery, 2015, 43 (08): 41-46.

[10] ZHANG J., GUO Y., GUO H., et al. Study on the Influence of Cooling Mode on the Performance of PDC Matrix Bit Body [J]. China Equipment Engineering, 2018 (16): 166-167.

[11] YANG J., CHEN W., HUANG Y. Study of the Influence of Pressure Mode on the Performance of Diamond Drill Bit [J]. Superhard Material Engineering, 2015, 27 (03): 6-10.

[12] SUN X., WANG J., LI K., et al. Influence of Temperature on PDC Bit Quality Analysis and its Controlling Measures [J]. Geotechnical Equipment, 2016, 17 (02): 18-23.

[13] WANG S., XIE D., NING C., et al. Influence of Particle Size Ratio on the Performance of WC-base Diamond Drill Bit [J]. Super hard Material Engineering, 2014, 26 (05): 9-12.

[14] Effect of Cast Tungsten Carbide Powders on Microstructure and Mechanical Properties of Polycrystalline Diamond Compact Bit Matrix [J]. Acta Materiae Compositae Sinica, 2019, 36 (05): 1235-1243.

[15] A. Shafiei, M. B. Dusseault, Geomechanical Properties of a Conglomerate from Iran [J]. ARMA 08-053

[16] YU Y., ZHANG G., LI Study on the Influence of Conglomerate Meso-structures Characteristics on Crack Propagation. ARMA 19-1742

[17] TENG X., CHEN M., YANG P., et al. Research and Application of ROP Enhancement Technology for in Kuqa Foreland Ultra-deep Reservoir Drilling [J]. China Petroleum Machinery, 2016, 21 (01): 76-88.

[18] WANG, W. Design and Test of a New PDC Bit with Tapered Auxiliary Cutter for Heterogeneous Formations [J]. Petroleum Drilling Techniques, 2018, 46 (02): 58-62.

[19] LIU Z, HOU H, HU W. An Experimental and Numerical Simulation Study on Rock Cutting Based on Pre-Breaking [J]. China Petroleum Machinery, 2019, 47 (12): 38-43+57.

[20] LIU J., HUANG L., DONG J., et al. Research on the rock breaking mechanism of PDC Bit [J]. Computer Era, 2019 (06): $5-8$.

[21] SUNW., ZHAO H., ZHANG T., et al. Experimental Study on Impact Resistance Enhancement of PDC Cutter [J]. Drilling \& Production Technology, 2018, 41 (06): 87-89. 